\title{
Malaria in Pregnancy and the Utilization of Preventive Interventions among Pregnant Women in Uyo, Nigeria
}

\section{Edidiong S Akpan' ${ }^{1}$, Dennis N Aribodor ${ }^{1}$, Ifeoma K Ugwuanyi ${ }^{1,2}$, Ogechukwu B Aribodor ${ }^{3}$, Ngozi N Joe-Ikechebelu ${ }^{4,5 *}$ and Amaechi C Nwachukwu $^{6,7}$}

${ }^{1}$ Department of Parasitology and Entomology, Nnamdi Azikiwe University, Awka, Anambra State, Nigeria

${ }^{2}$ School of Biological, Earth and Environmental Sciences, University of New South Wales, Sydney, Australia

${ }^{3}$ Department of Zoology, Nnamdi Azikiwe University, Awka, Anambra State, Nigeria ${ }^{4}$ Department of Community Medicine/Primary Health Care, Chukwuemeka Odumegwu Ojukwu University and Teaching Hospital, Amaku, Awka, Anambra State, Nigeria ${ }^{5}$ Social Dimensions of Health Program, School of Public Health and Social Policy, Human and Social Development, University of Victoria, British Columbia, Canada ${ }^{6}$ Department of Surgery, Chukwuemeka Odumegwu Ojukwu University, Amaku, Awka, Anambra State, Nigeria

${ }^{7}$ Chukwuemeka Odumegwu University Teaching Hospital, Amaku, Awka, Anambra

*Corresponding Author: Ngozi N Joe-Ikechebelu, Social Dimensions of Health Program, School of Public Health and Social Policy, Human and Social Development, University of Victoria, British Columbia, Canada. E-mail: ngozijoseph13@gmail.com
Received: May 24, 2021

Published: June 11, 2021

(C) All rights are reserved by Edidiong $\mathbf{S}$

Akpan., et al.

\begin{abstract}
Background and Objectives: Pregnancy-associated malaria remains a major risk to pregnant woman and her foetus in sub-Saharan Africa. Intermittent Preventive Treatment (IPT) and insecticide treated nets (ITNs) are the recommended malaria in pregnancy preventive interventions in the region. This cross-sectional study examined the prevalence of malaria in pregnant women. We also aimed to determine the use of these pregnant women regarding the recommended prevention interventions to understand barriers to uptake and help to improve their effectiveness.

Methods: Blood screening for malaria parasite was conducted on 405 pregnant women from Uyo in southern Nigeria. Information the on use of preventive measures were also obtained using questionnaires

Results: Our findings show that 105/405 (25.9\%) women were infected with malaria. On the use of preventive measures for malaria control, there was an above average compliance by the study population. For the use of the recommended IPT drug, 73.8\% of the women had taken a complete dose, while $25.9 \%$ failed to take the drug. Results also revealed that $68.3 \%$ of the women always slept under a Long-lasting Insecticide-treated Nets (LLINs) while $19.2 \%$ did not use the nets.

Interpretation and Conclusion: Our study indicates that malaria in pregnancy is still a problem in the study area. Therefore. advocacy on knowledge and behavior change practices should be strengthened to effectively prevent malaria for improved quality of life during and after pregnancy.

Keywords: Malaria; Pregnancy; IPT; LLIN
\end{abstract}

\section{Introduction}

Malaria, a mosquito-borne febrile illness, caused by the parasite Plasmodium parasite, continues to constitute a major source of morbidity and mortality globally. Although much progress has been made towards reducing malaria morbidity and mortality over the last century, an estimated 228 million cases occurred worldwide in 2018, leading to 405,000 deaths [1]. Most of the ma- laria morbidity were in the African region (93\%) followed by the South-East Asia region (3.4\%) and Eastern Mediterranean region (2.1\%) [1]. In malaria endemic areas, pregnant women are particularly susceptible to malaria [2] and is a major public health concern in tropical and subtropical countries. The high vulnerability of pregnant women to malaria infection is the result of the reduced immune response associated with pregnancy, coupled with the 
unique ability of a subset of $P$. falciparum infected erythrocytes to sequester in the placenta [3]. Infection of malaria during pregnancy poses a particular threat to pregnant women, with adverse outcomes such as malaria maternal anaemia, stillbirths, preterm labour, low birthweight and reduced intrauterine fetal growth [4].

In areas with stable malaria transmission, the prevalence of malaria in pregnancy ranges from $10 \%$ to $65 \%$ [5]. Outside Africa, $\boldsymbol{P}$. vivax infections are predominant, and every year about 90 million pregnant women are exposed to the risk of infection mostly in the Asia-Pacific region only, and about three million in Latin America [3]. Every year, approximately 50 million women living in malaria endemic areas become pregnant, and in sub-Saharan Africa, malaria affects an estimated 24 million pregnant women and hence this region records the greatest severity of malaria, accounting for $90 \%$ of all the deaths [6]. It has been reported that in sub-Saharan Africa, malaria can cause as many as 10,000 cases of malaria-related deaths in pregnancy per year, usually due to severe maternal anaemia [7]. Moreover, each year, malaria in pregnancy is responsible for $20 \%$ of stillbirths, and $11 \%$ of all newborn deaths in subSaharan Africa [8].

Malaria in pregnancy remains a major public health challenge in Nigeria, with a quarter of the burden in Africa occurring in the country [9]. Moreover, over $12.0 \%$ of the gross domestic product (GDP) is expended on malaria annually in the country [10]. Current estimates of malaria parasitaemia in Nigerian pregnant women show great disparity and vary considerably among geographic regions. Hospital-based prevalence ranges from $5 \%$ in the northwestern region [11], $19.7 \%$ to $40.2 \%$ in the southwestern region [12,13], $66.4 \%$ to $95 \%$ in the southeastern region $[14,15]$. In south-west Nigeria, past studies reported malaria parasite prevalence in pregnancy to be of the range of $7.7 \%$ [16] to $60 \%-72 \%$ $[17,18]$. Bassey., et al. [19] in a study done in Port Harcourt, southern Nigeria, estimated that the prevalence of placental malaria was $65.2 \%$. In a community-based study to estimate the burden of malaria in pregnancy in Enugu, southeast Nigeria, Gunn., et al. [20] noted that of the 2069 pregnant women examined, over $99 \%$ were positive for malaria parasitaemia. In north-western Nigeria, Fana., et al. [21] estimated a malaria prevalence rate of $41.6 \%$ out of 255 pregnant women.

Successful control of malaria which involves preventing the infection as well as treatment in pregnant women is a major step in curbing the burden of malaria in Africa [22]. In view of this, preventive measures recommended by World Health Organization for Africa include maintaining a clean environment, use of insecticide treated nets (ITN), intermittent preventive treatment in pregnancy and effective case management of both complicated and uncomplicated cases [23]. The Nigeria National Malaria Control Programme [24] exclusively recommends 2 doses of Sulphadoxine-pyrimethamine for chemoprevention of malaria during normal pregnancy. Another current effort to address malaria illness in pregnancy is the use of Long-lasting Insecticide-treated bednets (LLINs) to prevent contact with the mosquito vector [25]. Sleeping under insecticide-treated bednets has not only remained one of the most important of all measures of protecting pregnant women against malaria, it has become the single most dependable intervention when used properly and efficacious in reducing maternal anaemia, placental infection, and low birth weight [26].

Despite the impressive reductions in malaria incidence recorded in Nigeria over the past 15 years as a result of the aforementioned intervention strategies, as seen in the 2015 World Malaria Report [6], malaria mortality reduction rate has also slowed since 2015 as reflected in the same report released in 2018 [8]. However, the management and control of malaria in pregnancy in Nigeria is impaired by the inconsistency of local prevalence statistics. It therefore remains of critical importance to determine the prevalence of malaria as well as to better understand the prevention practices among pregnant women in the study population. Results from this study would provide baseline data which could be integrated into the existing State and National data on malaria in pregnancy control programme. The data will allow for the improvement and development of strategies targeting malaria prevention and treatment in this population. Such control measures may also possibly lead to reduction in prevalence, which is geared towards achieving malaria elimination in Nigeria.

\section{Materials and Methods}

Ethical statement

Ethical approval was granted by the Health Research Ethics Committee of the Akwa-Ibom State Ministry of Health. Verbal informed and free consent was obtained from the participants. The participants were informed that participation in the research was voluntary. The pre-tested questionnaires were administered by trained interviewers who interpreted the contents and the consent forms to those with poor understanding of English in local dialects. Those below the age of 18 years had their accompanying husband or older relatives consent for them.

\section{Study site}

The study was carried out in Uyo Local Government Area of Akwa Ibom State in South-south Nigeria. Malaria is holoendemic in 
Akwa Ibom State. Uyo is characterised by a tropical humid climate without distinct seasonal variation. It has annual precipitation of about $1000 \mathrm{~mm}$, though December to February has considerably low rainfall, and little variability in temperature [27]. Uyo Local Government Area is made up of four communities namely: Offot, Etoi, Oku and Ikono. Four hospitals were selected to represent each of these communities and cover private and public facilities. The hospitals were: St. Luke's hospital Anua, Offot, Primary Health Care Center Ikono, Primary Health Care Center Oku, and Dan-Abia Specialist Hospital Etoi.

\section{Study population}

The study was carried out over a period of November 2017 to May 2018. Pregnant women who attended antenatal care and consented to participate in the study during this period were included. It is important to state that only the female population of reproductive age (15 - 49 years) who make up $49 \%$ of the total female population were involved in the study. Questionnaires were administered to the participants to obtain information about their socio-demographic characteristics, ITN usage, IPT usage and pregnancy history.

\section{Sample size}

A total of 405 (greater than the determined sample size, by 5) pregnant women were sampled for prevalence of malaria in pregnancy, IPT, and use of ITNs. The population of the study community was based on the 2006 Nigerian Census as updated up to 2016 and was used to determine the sample size of this study. Within the study population, only $5 \%$ of the women of reproductive age are considered pregnant.

The sample size (n) was determined from the total population of Uyo: 429,900 using Taro Yamane's formula [28] for determining a sample size from a finite population.

\section{Determination of malaria parasitaemia}

About $2 \mathrm{mls}$ of venous blood was obtained from a peripheral vein of each participant and transferred into an EDTA bottle. Each sample was labelled correctly with the patient's personal data to avoid any mix up. A total of 405 samples of blood specimens were collected. Thick and thin film microscopy of the peripheral blood for the confirmation of the presence or absence of malaria parasite followed the methods of Cheesbrough [29] and Sood [30]. The prepared blood smears were air dried and subsequently stained with freshly prepared Giemsa stain at $\mathrm{pH}$ 7.2. The stained smears were examined under $\times 100$ oil immersion lens of a light microscope. Malaria diagnosis was based on identification of asexual stages of
Plasmodium species on the thick blood film while thin smears were used for species identification. Parasite density was determined by counting the number of parasites per high power field and ranged from $+(1-10$ parasites per 100 high power fields $),++(>10$ parasite per 100 high power fields), +++ (1 - 10 parasites in one high power field), and ++++ ( $>10$ parasites in one high power field). The slides were reported as negative if no parasite was identified per 100 power fields.

Determination of preventive measures among pregnant women

Structured questionnaire (405) designed to elicit information on the use of ITNs, intake of IPT, reasons for not complying with the usage, and frequency of use was issued to pregnant women on their ante-natal care (ANC) visits. The questionnaire was administered to pregnant women who could read and write and self-administered to pregnant women who could neither read nor write.

\section{Analysis of data}

IBM SPSS Version 26 Statistical software was used to analyze the data. Prevalence and use of preventive measures were analyzed using the frequency and percentage, while $\chi^{2}$ (Chi-square) to determine any statistical differences between the variables at $5 \%$ and $1 \%$ levels of significance, and at specified degrees of freedom.

\section{Results}

Prevalence of malaria parasites of pregnant women by age

A total of 405 pregnant women between the ages of $16-46$ years participated in the study. Malaria prevalence among this population was $25.9 \%$ (105/405). Highest prevalence of $80.0 \%$ was observed in the age group 16 - 20 years and the least prevalence of $14.2 \%$ was recorded for the age group 41-46 years. Age was significantly associated with malaria prevalence $(p=0.001)$ (Figure 1).

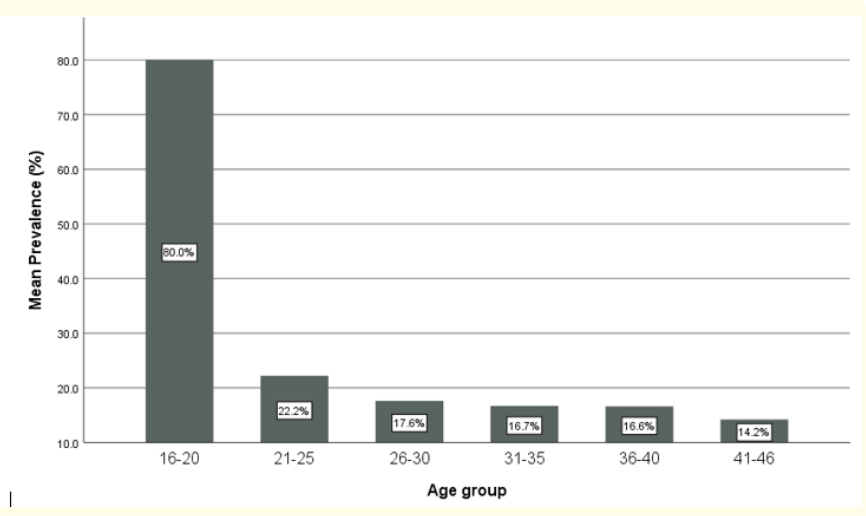

Figure 1: Prevalence of malaria among pregnant women in relation to age. 
Frequency of ITN use by respondents

A total of 277 (68.3\%) respondents use LLINs daily to prevent mosquito bites. Conversely, $19.2 \%$ reported that they do not use LLINs (Table 1).

\begin{tabular}{|c|c|c|}
\hline Frequency & No. & \% \\
\hline Used daily & 277 & 68.3 \\
\hline Occasionally & 50 & 12.3 \\
\hline Never & 78 & 19.2 \\
\hline Total & 405 & 100 \\
\hline
\end{tabular}

Table 1: Use of LLIN by pregnant women in Uyo, Akwa Ibom State.

Of the 128 who reported they either used LLINs occasionally or were not using it at all, 46.0\% (59/128) reported that LLIN generates heat, $28.9 \%(37 / 128)$ opined that they had difficulty setting it up, $15.6 \%(20 / 128)$ claimed they use insecticides instead of LLINs and 9.3\% (12/128) claimed there was no mosquitoes in their houses as shown in table 2.

\begin{tabular}{|c|c|c|}
\hline Reasons for non-usage/sometimes usage & No. & $\mathbf{\%}$ \\
\hline Heat generation & 59 & 46.0 \\
\hline Difficulty in setting up & 37 & 28.9 \\
\hline Use of Insecticide & 20 & 15.6 \\
\hline No mosquito in my house & 12 & 9.3 \\
\hline Total & 128 & \\
\hline
\end{tabular}

Table 2: Reasons for non-usage of LLINs by pregnant women.

Table 3 shows the relationship between the parity of the women and the usage of LLINs. Of the 63 malaria-positive primigravidae mothers, $4.7 \%$ (3/63) used LLINs daily while 61.9\% (39/63) did not use at all. Also, out of 17 multigravidae mothers infected with malaria, 23.5\% (4/17) used LLINs daily while 64.7\% (11/17) did not use LLINs at all.
Of the 21 malaria-negative primigravidae mothers, $80.0 \%$ $(17 / 21)$ used LLINs daily while $4.7 \%(1 / 21)$ did not use LLINs at all. Also, out of 103 malaria-negative multigravidae mothers, 86.4\% (89/103) used LLINs daily and 4.9\% (5/103) did not use at all LLINs at all, as shown in table 4 . There was a statistical difference $(\mathrm{P}=0.019)$ among pregnant women who used LLINs and those who did not make use of it.

On the use of IPT for malaria, the result showed that $73.8 \%$ (299/405) received of IPT, while $0.2 \%(1 / 405)$ took incomplete dosage and 25.9\% (105/405) did not take IPT as shown in table 5.

Various reasons were given for not receiving IPT as shown on table 6. Among the study participants, $15.3 \%(62 / 105)$ opined that taking IPT may result in abortion, $0.2 \%(1 / 105)$ reported bleeding from the first dose and $10.3 \%$ (43/105) said they were not sick at the moment.

Associating malaria status of pregnant women with intake of IPT (Table 7), the result showed that of the 63 malaria-positive primigravidae mothers, 1.6\% (1/63) took complete dose of IPT and $98.4 \%(62 / 63)$ did not take IPT. Also, of the 17 malaria-positive multigravidae mothers, $5.9 \%$ (1/17) took incomplete dose and 94.1\% (16/17) did not take IPT.

On the pregnant women who tested negative for malaria parasite, all the 21 malaria negative primigravidae mothers took complete dose of IPT. Out of 103 malaria negative multigravidae mothers, $97.1 \%$ (100/103) took complete dose while 2.9\% (3/103) did not take IPT as seen in table 8 . There was a significant difference $(\mathrm{P}=0.001)$ among pregnant women who took complete dosage, incomplete dosage and pregnant women who did not take.

\begin{tabular}{|c|c|c|c|c|c|}
\hline Parity & $\begin{array}{c}\text { No. } \\
\text { Examined }\end{array}$ & $\begin{array}{c}\text { No. +ve } \\
\text { for malaria } \\
\text { parasite }\end{array}$ & $\begin{array}{c}\text { No. of women who used } \\
\text { LLINs daily and tested } \\
\text { positive }\end{array}$ & $\begin{array}{c}\text { No. of women who } \\
\text { used LLINs occasion- } \\
\text { ally and are positive }\end{array}$ & $\begin{array}{c}\text { No. of women who did not } \\
\text { use LLINs and tested } \\
\text { positive }\end{array}$ \\
\hline Primigravidae & 84 & 63 & $3(4.7 \%)$ & $21(33.3 \%)$ & $39(61.9 \%)$ \\
\hline Secundigravidae & 201 & 25 & $2(8.0 \%)$ & $3(12.0 \%)$ & $20(80.0 \%)$ \\
\hline Multigravidae & 120 & 17 & $4(23.5 \%)$ & $2(11.8 \%)$ & $11(64.7 \%)$ \\
\hline Total & 405 & 105 & 9 & 26 & 70 \\
\hline
\end{tabular}

Table 3: Relationship between parity and usage of LLIN among malaria infected respondents. 


\begin{tabular}{|c|c|c|c|c|c|}
\hline Parity & No. Examined & $\begin{array}{c}\text { No. negative } \\
\text { for malaria } \\
\text { parasite }\end{array}$ & $\begin{array}{c}\text { No. of women who } \\
\text { used LLINs daily and } \\
\text { tested negative }\end{array}$ & $\begin{array}{c}\text { No. of women } \\
\text { who used LLINs } \\
\text { occasionally and } \\
\text { are negative }\end{array}$ & $\begin{array}{c}\text { No. of women who } \\
\text { did not use LLINs and } \\
\text { tested } \\
\text { negative }\end{array}$ \\
\hline Primigravidae & 84 & 21 & $17(80.0 \%)$ & $3(14.2 \%)$ & $1(4.7 \%)$ \\
\hline Secundigravidae & 201 & 176 & $162(92.0 \%)$ & $12(6.8 \%)$ & $2(1.1 \%)$ \\
\hline Multigravidae & 120 & 103 & $89(86.4 \%)$ & $9(8.7 \%)$ & $5(4.9 \%)$ \\
\hline Total & 405 & 105 & 268 & 24 & 8 \\
\hline
\end{tabular}

Table 4: Relationship between parity and usage of LLIN among malaria negative respondents.

\begin{tabular}{|c|c|c|}
\hline Receipt of IPT & No. of pregnant women & \% \\
\hline Completed dose & 299 & 73.8 \\
\hline Incomplete dose & 1 & 0.2 \\
\hline Not taken & 105 & 25.9 \\
\hline Total & 405 & \\
\hline
\end{tabular}

Table 5: Use of IPT among pregnant women in Uyo, Akwa Ibom State.

\begin{tabular}{|c|c|c|}
\hline Reason & No. of respondents & $\mathbf{\%}$ \\
\hline Fear of Abortion & 62 & 59.0 \\
\hline Had bleeding from the first dose & 1 & 0.95 \\
\hline Not sick at the moment & 43 & 40.9 \\
\hline Total & 105 & \\
\hline
\end{tabular}

Table 6: Reasons for not taking IPT among pregnant women.

\begin{tabular}{|c|c|c|c|c|c|}
\hline Parity & No. Examined & $\begin{array}{c}\text { No. positive } \\
\text { for malaria } \\
\text { parasite }\end{array}$ & $\begin{array}{c}\text { No. of women who } \\
\text { took complete IPT } \\
\text { and tested positive }\end{array}$ & $\begin{array}{c}\text { No. of women who took } \\
\text { incomplete IPT dose and } \\
\text { tested positive }\end{array}$ & $\begin{array}{c}\text { No. of women who } \\
\text { did not use IPT and } \\
\text { tested positive }\end{array}$ \\
\hline Primigravidae & 84 & 63 & $1(1.6 \%)$ & 0 & $62(98.4 \%)$ \\
\hline Secundigravidae & 201 & 25 & $2(8.0 \%)$ & 0 & $23(92.0 \%)$ \\
\hline Multigravidae & 120 & 17 & 0 & $1(5.9 \%)$ & $16(94.1 \%)$ \\
\hline Total & 405 & 105 & 3 & 1 & 101 \\
\hline
\end{tabular}

Table 7: Relationship between parity and intake of IPT among malaria infected respondents.

\begin{tabular}{|c|c|c|c|c|c|}
\hline Parity & No. Examined & $\begin{array}{c}\text { No. negative } \\
\text { for malaria } \\
\text { parasite }\end{array}$ & $\begin{array}{c}\text { No. of women who took } \\
\text { complete IPT dose and } \\
\text { tested negative }\end{array}$ & $\begin{array}{c}\text { No. of women who took } \\
\text { incomplete IPT dose and } \\
\text { tested positive }\end{array}$ & $\begin{array}{c}\text { No. of women who } \\
\text { did not use IPT and } \\
\text { tested negative }\end{array}$ \\
\hline Primigravidae & 84 & 21 & 21 & 0 & 0 \\
\hline Secundigravidae & 201 & 176 & $175(99.4 \%)$ & 0 & $1(0.6 \%)$ \\
\hline Multigravidae & 120 & 103 & $100(97.1 \%)$ & 0 & $3(2.9 \%)$ \\
\hline Total & 405 & 300 & 296 & 0 & 4 \\
\hline
\end{tabular}

Table 8: Relationship between parity and intake of IPT among malaria-negative respondents.

\section{Discussion}

A prevalence of $25.9 \%$ for malaria in pregnancy was observed among the pregnant women. This rate represents a decrease in prevalence compared to $54.7 \%$ and $41.0 \%$ of maternal malaria infection earlier reported by Ikpeze., et al. [31] from the same study area. The observed reduction may be due to the improved use of malaria preventive measures including LLIN and IPT by pregnant women.
The highest prevalence of $40.0 \%$ observed in the age group 16-20 years agrees with the findings of Agomo., et al. [32] who reported that the highest malaria prevalence was seen in pregnant women $<20$ years in Lagos [16]. This finding could be attributed to the fact that immunity to malaria is lower in younger age groups, but as age increases, frequent exposure to malaria creates a stronger immunity, hence resulting in lower prevalence among older age groups [33]. Eneanya [34] also noted that the people in the younger 
age group had the highest prevalence of malaria because they have less knowledge on malaria prevention practices such as preventing exposure to bites of malaria vectors. As a consequence, this subset of the population exhibits a carefree lifestyle which includes exposing themselves to those vectors [35].

From this study, prevalence of malaria was found to be higher in primigravidae (72.6\%) compared to secundigravidae (13.9\%) and multigravidae (13.3\%). This agrees with the works of Nwachukwu and Chinemerem [36], Aribodor., et al. [15] and Ikpeze., et al. [31], in which highest prevalence was found among pregnant women in the primigravidae followed by multigravidae and least in the control group. It has previously been documented that immunity is gradually acquired following consecutive pregnancies, and this plays an important role in controlling malaria infection during pregnancy [37]. Mbanefo., et al. [38] also reported that women in their first pregnancy are most vulnerable to malaria infection. Such immunity is thought to arise either from the priming of memory T-cells or from the production of antibodies against $P$. falciparum chondroitin sulfate A (PfCSA) that prevents parasite sequestration in the placenta $[39,40]$.

On responses to the usage and reasons for non-usage of LLINs, $68.3 \%$ of the pregnant women opined that they used LLINs daily. Similarly, $73.8 \%$ of pregnant women took a complete dosage of IPT. This represents a remarkable improvement when compared with the study of Ikpeze., et al. [31] who reported $46.2 \%$ for LLINs usage and $48.1 \%$ for intake of IPT. This improvement could be attributed to improved public enlightenment on the use of these malaria preventive tools during pregnancy in Akwa-Ibom State as evidenced in the daily radio jingles in both local and English languages circulated in the state. The current ongoing public health campaign which involves free distribution of LLINs to pregnant women during antenatal visits could also account for the high rate of LLINs usage observed in this study. Gamble., et al. [41] and Adeyemi., et al. [42] have shown that LLIN use in pregnancy reduced the incidence of malaria in pregnancy, and hence the incidence of pregnancy-related complications such as premature deliveries, low birth-weight babies, maternal anaemia and intra-uterine foetal deaths.

\section{Conclusion}

Malaria prevalence at $25.9 \%$ among pregnant women, though a reduction from previous comparatives studies, is still high, as malaria prevalence takes into consideration population growth. This implies that as the population size rises, prevalence rate is likely to increase and as such needs to be addressed as Nigeria moves towards elimination. The implication of this fact is that malaria in pregnancy is still a public health problem in the study area. The usage of LLINs and IPT also recorded an improvement from past studies, but still falls below national target of $80 \%$, thus emphasizing the need for intensified efforts. The findings of this study are an indication that malaria in pregnancy is still a major issue in $\mathrm{Ni}$ geria. Nigerians especially pregnant women should be enlightened on the effectiveness of the use of IPT and LLINs for prevention of malaria. Efforts should also be geared towards improving availability, affordability and adaptability of control measures especially in the resource-constrained settings. Political will, increased investment in interventions and a more intensified research in this area is strongly advocated.

\section{Acknowledgments}

We are grateful to the journal editors and reviewers for their constructive comments. We are also grateful to the hospital staff for their assistance during the study, and the participants for their voluntary participation in this study.

\section{Disclosure Statement}

No potential conflict of interest was reported by the authors.

\section{Source of Funding}

Researchers have used own finance to complete research study.

\section{Bibliography}

1. World Health Organization. "World malaria report 2019". Published online (2019): 232.

2. Desai M., et al. "Epidemiology and burden of malaria in pregnancy". Lancet Infectious Disease 7.2 (2007): 93-104.

3. Rijken MJ., et al. "Malaria in pregnancy in the Asia-Pacific region”. Lancet Infectious Disease 12.1 (2012): 75-88.

4. Corrêa G., et al. "High burden of malaria and anemia among tribal pregnant women in a chronic conflict corridor in India". Conflict of Health 11.1 (2017): 1-9.

5. Pell C., et al. "Prevention and management of malaria during pregnancy: findings from a comparative qualitative study in Ghana, Kenya and Malawi”. Malar Journal 12.1 (2013): 427.

6. World Health Organization. "World Malaria Report 2015". World Health Organization 2015. Accessed January 31, (2020). 
7. Steketee RW., et al. "The burden of malaria in pregnancy in malaria-endemic areas". American Society of Tropical Medicine and Hygiene 64 (2001): 28-35.

8. World Health Organization. WHO | Implementing malaria in pregnancy programs in the context of World Health Organization recommendations on antenatal care for a positive pregnancy experience. WHO. Published 2018. Accessed February 6, (2020).

9. Olasehinde GI., et al. "Prevalence of malaria and predisposing factors to anti-malaria drug resistance in south-western $\mathrm{Ni}$ geria". (2014).

10. Jimoh A., et al. "Quantifying the economic burden of malaria in Nigeria using the willingness to pay approach". Cost Effectiveness and Resource Allocation 5 (2007): 6.

11. Isah AY., et al. "Prevalence of malaria parasitemia amongst asymptomatic pregnant women attending a Nigerian teaching hospital”. Annals of African Medicine 10.2 (2011): 171-174.

12. Simon-Oke IA. "Prevalence of Malaria Parasites among Pregnant Women and Children under Five years in Ekiti State, Southwest Nigeria". Journal of Biomedical and Translational Research 5.1 (2019): 5-10.

13. Uneke CJ., et al. "Assessment of malaria in pregnancy using rapid diagnostic tests and its association with HIV infection and hematologic parameters in South-Eastern Nigeria". Haematologica 93.1 (2008): 143-144.

14. Agan T., et al. "Prevalence of anemia in women with asymptomatic malaria parasitemia at first antenatal care visit at the University of Calabar Teaching Hospital, Calabar, Nigeria". International Journal of Women's Health 2 (2010): 229-233.

15. Dn A., et al. "Association of low birth weight and placental malarial infection in Nigeria". Journal of Infection in Developing Countries 3.8 (2009): 620-623.

16. Agomo CO., et al. "Prevalence of Malaria in Pregnant Women in Lagos, South-West Nigeria". Korean Journal of Parasitology 47.2 (2009): 179-183.

17. Adefioye 0., et al. "Prevalence of Malaria Parasite Infection among Pregnant Women in Osogbo, Southwest, Nigeria". (2006): 2 .
18. Okwa 00. "The status of malaria among pregnant women: a study in Lagos, Nigeria". African Journal of Reproductive Health 7.3 (2003): 77-83.

19. Bassey G., et al. "Prevalence of placenta Plasmodium parasitemia and pregnancy outcome in asymptomatic patients at delivery in a University Teaching Hospital in Nigeria". Nigerian Journal of Clinical Practice 18.1 (2015): 27.

20. Gunn JKL., et al. "Population-based prevalence of malaria among pregnant women in Enugu State, Nigeria: the Healthy Beginning Initiative". Malaria Journal 14.1 (2015): 438.

21. Fana SA., et al. "Prevalence and risk factors associated with malaria infection among pregnant women in a semi-urban community of north-western Nigeria". Infectious Diseases of Poverty 4 (2015): 24

22. Aribodor D., et al. "Challenges to achieving malaria elimination in Nigeria". American Journal of Public Health 4.1 (2016): 3841.

23. World Health Organization. Global health estimates 2016. "Disease burden and mortality estimates". Published (2016).

24. National Malaria Control Programme. "National Malaria Strategic Plan 2009-2013". Published online (2009).

25. Federal Ministry of Health. "National integrated community case management of malaria”. (2012): 1.

26. WHO/UNICEF. "Lives at risk: malaria in pregnancy". WHO. Published (2003).

27. Meteoblue. "Climate Uyo". Meteoblue (2019).

28. Yamane T. “Statistics: An Introductory Analysis” (1967).

29. Cheesbrough M. "Examination of blood for malaria parasites". In: District Laboratory Practice in Tropical Countries Part 1. Cambridge Low-Price Edition (2006): 239-258.

30. Sood R. "Textbook of Medical Laboratory Technology". First. Jaypee Medical Publisher (2006).

31. Ikpeze 0., et al. "Effects of non-compliance to LLIN and IPT in pregnancy on maternal and neonatal malaria, age and birth weights of neonates at Uyo". International Journal of Research GRANTHAALAYAH 4 (2016): 66-74. 
32. Agomo CO., et al. "Prevalence of Malaria in Pregnant Women in Lagos, South-West Nigeria". Korean Journal of Parasitology 47.2 (2009): 179-183.

33. Askling HH., et al. "Malaria Risk in Travelers". Emerging Infectious Diseases 11.3 (2005): 436-441.

34. Eneanya C. "Seasonal variation in malaria episodes among residents in a semi-urban Community in South-East Nigeria". Nigerian Journal of Parasitology 19 (1998): 39-43.

35. Eneanya CI. "Seasonal Variation in Malaria Episodes among Residents of Udi, a Semi-Urban Community in Southeast Nigeria". Nigerian Journal of Parasitology 19.1 (1998): 39-43.

36. Nwachukwu E and Chinemerem O. "Evaluation of the use of intermittent preventive therapy and long-lasting insecticidal nets for malaria prevalence among pregnant women in Nkpor, Nigeria". Pharmacy and Pharmacology International Journal 6.1 (2018).

37. Uneke CJ., et al. "Assessment of malaria in pregnancy using rapid diagnostic tests and its association with HIV infection and hematologic parameters in South-Eastern Nigeria". Haematologica 93.1 (2008): 143-144.

38. Mbanefo E., et al. "Antenatal Malaria Parasitaemia and Haemoglobin Profile of Pregnant Mothers in Awka, Anambra State, Southeast Nigeria". American-Eurasian Journal of Agricultural and Environmental Sciences 4 (2009): 235-239.

39. Maubert B., et al. "Development of antibodies against chondroitin sulfate A-adherent Plasmodium falciparum in pregnant women". Infection on Immunology 67.10 (1999): 53675371.

40. Moore JM., et al. "Immunologic memory in the placenta: a lymphocyte recirculation hypothesis". Medical Hypotheses 54.3 (2000): 505-510.

41. Gamble C., et al. "Insecticide-Treated Nets for the Prevention of Malaria in Pregnancy: A Systematic Review of Randomised Controlled Trials". PLOS Medicine 4.3 (2007): e107.

42. Adeyemi AS., et al. "Use Prevalence of Insecticide-Treated Mosquito Bed Nets among Pregnant Population in Osogbo, Nigeria". Nigerian Medical Practitioner 52.2 (2007): 29-32.

Volume 4 Issue 7 July 2021

C) All rights are reserved by Edidiong $S$ Akpan., et al. 